\title{
Beam Diagnostics Via Model Independent Analysis of the Turn-by-Turn BPM Data
}

\author{
Xi Yang \\ Fermi National Accelerator Laboratory \\ Box 500, Batavia IL 60510
}

\begin{abstract}
Model independent analysis (MIA) can be used to obtain all the eigen modes included in the turn-by-turn BPM data. Not only the synchrotron tune and betatron tune can be obtained from the fast Fourier transforms (FFT) of the temporal eigen vector of the corresponding mode, but also the error mode, which could be caused by the different gain of a BPM, can be observed in both the temporal and spatial eigen vectors of the error mode. It can be applied as a diagnostic tool for Booster.

\section{Introduction}

The turn-by-turn data from all the BPM can be used to form a BPM matrix $\left(B_{M \times N}\right)$. The single-turn data from all the BPM form a row in the matrix. And the column number is equivalent to the BPM number. Once the BPM matrix is formed, MIA can be used to obtain all the eigen modes, which form a complete basis of the BPM matrix.[1] Since the number of orbits $(M)$ is greater than the number of BPM $(N)$, the maximum number of eigen modes is determined by the BPM number. Only several eigen modes are considered to be significant to the orbit, and other modes are neglected as noises. Generally, two betatron modes and one synchrotron mode due to non-zero dispersions at BPM locations are observed.[1] If more modes are observed, they are generally caused by the incorrect gain of a BPM, or some other errors. So finding the cause of an unexpected mode can be used for the diagnostic purpose.
\end{abstract}

\section{Method}

Single value decomposition (SVD), as shown in eq.1, is used to calculate all the eigen modes and eigen vectors of the BPM matrix $(B)$.[2]

$B_{M \times N}=U_{M \times M} S_{M \times N}\left(V_{N \times N}\right)^{T}$. 
Each element $S_{i, i}$ of the $S$ matrix represents the eigen value of the $i^{\text {th }}$ eigen mode, and the eigen value decreases with the increase of the number $i$. The $i^{\text {th }}$ column of the $U$ matrix is the temporal eigen vector of the $i^{\text {th }}$ eigen mode, and $i^{\text {th }}$ row of the $V^{T}$ matrix is the spatial eigen vector of the $i^{\text {th }}$ eigen mode. MATLAB is used for the SVD decomposition of the BPM matrix.

\section{Simulation and Results}

The $n^{\text {th }}$ BPM reading at the $m^{\text {th }}$ turn $\left(x_{n}(m)\right)$ can be written as the summation of three different terms, as shown in eq.2.[3]

$x_{n}(m)=x_{c o}+x_{D}(m)+x_{\beta}(m)$

$=B_{m, n}$.

Eq. 2 was used to generate the $B$ matrix with the input of the lattice file from MAD.[4] $x_{c o}$ is set to be zero due to the consideration of the difference orbit. Also, the operational parameters were used in the calculation. They are the synchrotron tune $Q_{s}=0.05$, horizontal betatron tune $Q_{x}=6.7$, chromaticity $\xi=6$, the number of BPM $N=48$, and the number of tracking turns $M=128$. The beam was excited both transversely and longitudinally at the $0^{\text {th }}$ turn with a 1-mrad angle kick at the $1^{\text {st }}$ BPM and a momentum offset $\delta=0.005$. The $1^{\text {st }}$ turn and $128^{\text {th }}$ turn orbits are shown in Fig. 1(a) as the red and black curves separately. Eigen values of all the modes are shown in Fig. 1(b). There are three significant modes, and they are caused by the synchrotron motion and betatron motion of the beam. Their temporal eigen vectors are shown in Figs. 1(c), 1(e), and 1(g) for modes 1, 2, and 3 separately, and their corresponding spatial eigen vectors are shown in Figs. 1(d), 1(f), and 1(h). Their unnormalized power spectrums are shown in Figs. 1(i)-(k). Also, chromatic sidebands appear in the unnormalized power spectrum of both betatron modes, as shown in Fig. 1(j) and Fig. 1(k).[3]

The above simulation was repeated at different gains of the $1^{\text {st }}$ BPM. When the gain of the $1^{\text {st }}$ BPM is set to be 1.5, eigen values of all the modes are shown in Fig. 2(a). There are four significant modes, and they are the error BPM mode, synchrotron mode and two betatron modes. Eigen values of the synchrotron mode and two betatron modes in Fig. 2(a) are the same with those in Fig. 1(b), and they are much smaller than the eigen value of the BPM error mode, as shown in Fig. 2(a). The temporal eigen vector of mode 1 is 
shown in Fig. 2(b), its unnormalized power spectrum and spatial eigen vector are shown in Figs. 2(c) and (d) separately. Also, the eigen value of the BPM error mode changes with the gain of the $1^{\text {st }}$ BPM, as shown in Fig. 2(e), however, eigen values of the synchrotron mode and two betatron modes stay the same.

Instead the $1^{\text {st }}$ BPM has a different gain since the $1^{\text {st }}$ turn, it had a different gain from the $60^{\text {th }}$ turn. Eigen values of all the modes are shown in Fig. 3(a). The temporal eigen vector of the BPM error mode (mode 1) is shown in Fig. 3(b), its unnormalized power spectrum and spatial eigen vector are shown in Figs. 3(c) and (d). The eigen value of the BPM error mode changes with the gain of the $1^{\text {st }}$ BPM, as shown in Fig. 3(e).

\section{Conclusion}

From the power spectrum of the synchrotron mode and two betatron modes, the synchrotron tune and the betatron tune can be obtained. Also, whenever the gain of the BPM is set at a wrong value, an extra BPM error mode will be observed. The temporal eigen vector of the BPM error mode can be used to extract the time when the gain of the BPM starts to be wrong, as shown in Fig. 2(b) and Fig. 3(b), and the spatial eigen vector can be used to obtain the position where the BPM error is, as shown in Fig. 2(d) and Fig. $3(d)$.

\section{Acknowledgements}

The author thanks for the useful discussion with James MacLachlan and Jim Lackey, and also the encouragement from Chuck Ankenbrandt.

\section{References:}

[1] Chun-xi Wang, "Model Independent Analysis of Beam Centroid Dynamics in Accelerators", SLAC-547.

[2] www.mathworks.com.

[3] X. Yang, "Chromaticity Measurement Via the Fourier Spectrum of Transverse Oscillations", FERMILAB-TM-2262-AD, submitted.

[4] http://www-ap.fnal.gov/ drozhdin/prdriver/ 


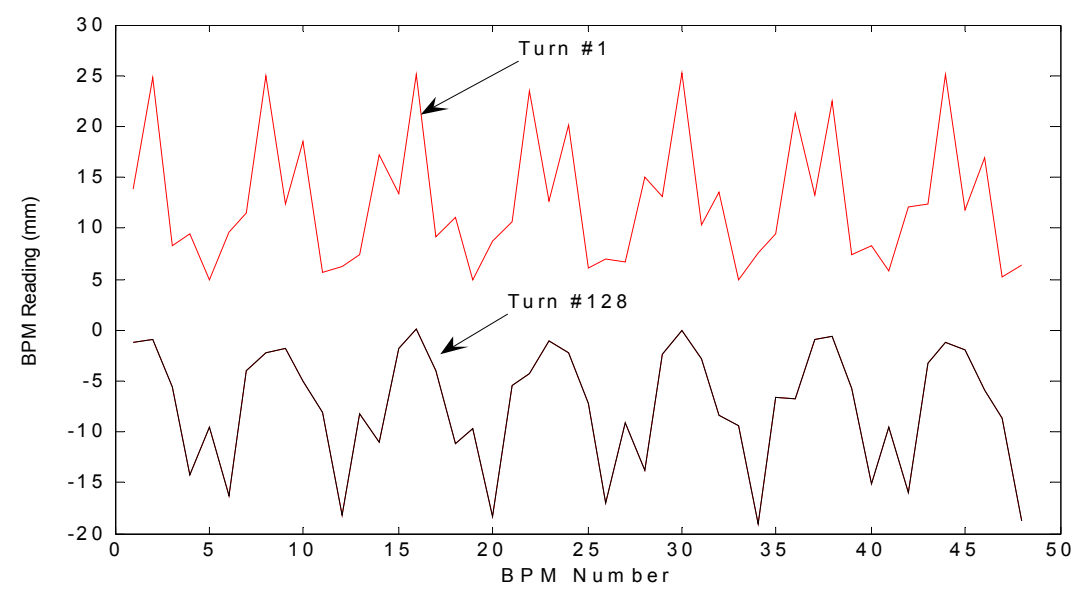

Fig. 1(a)

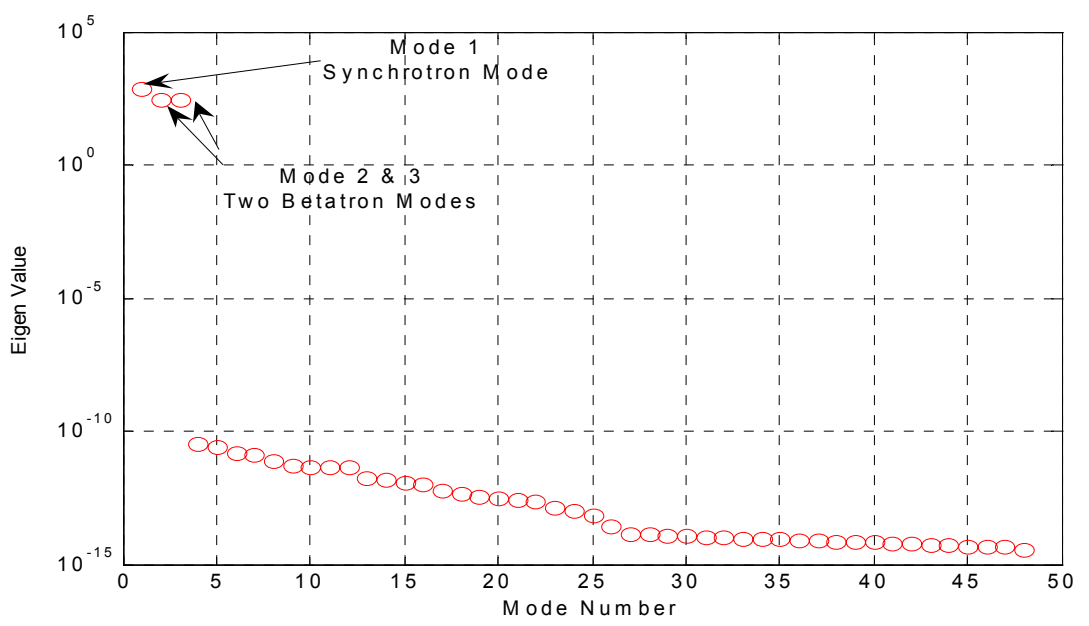

Fig. 1(b)

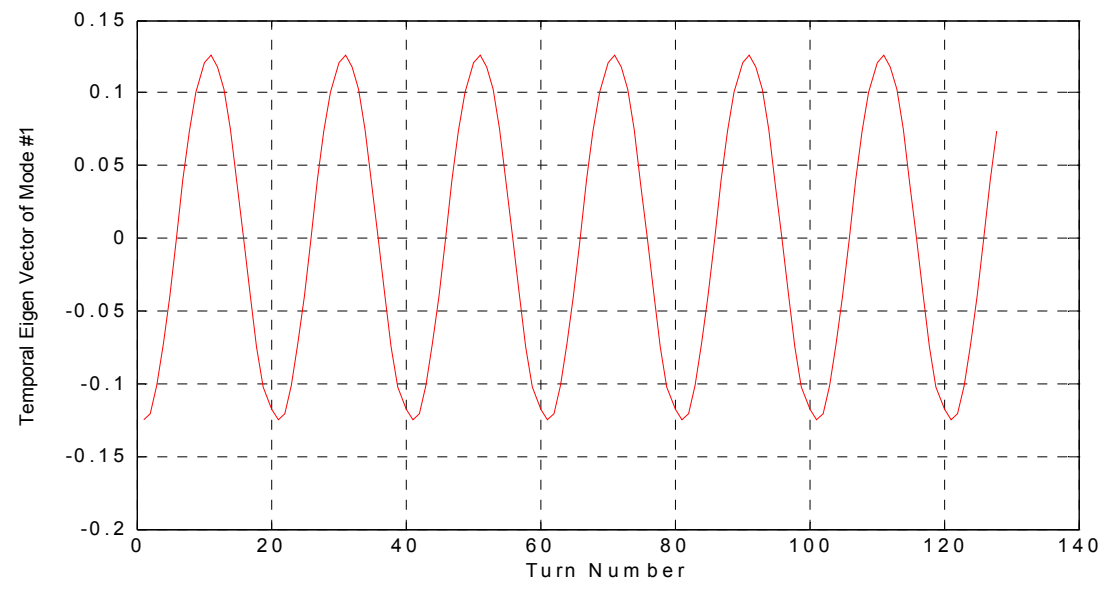

Fig. 1(c) 


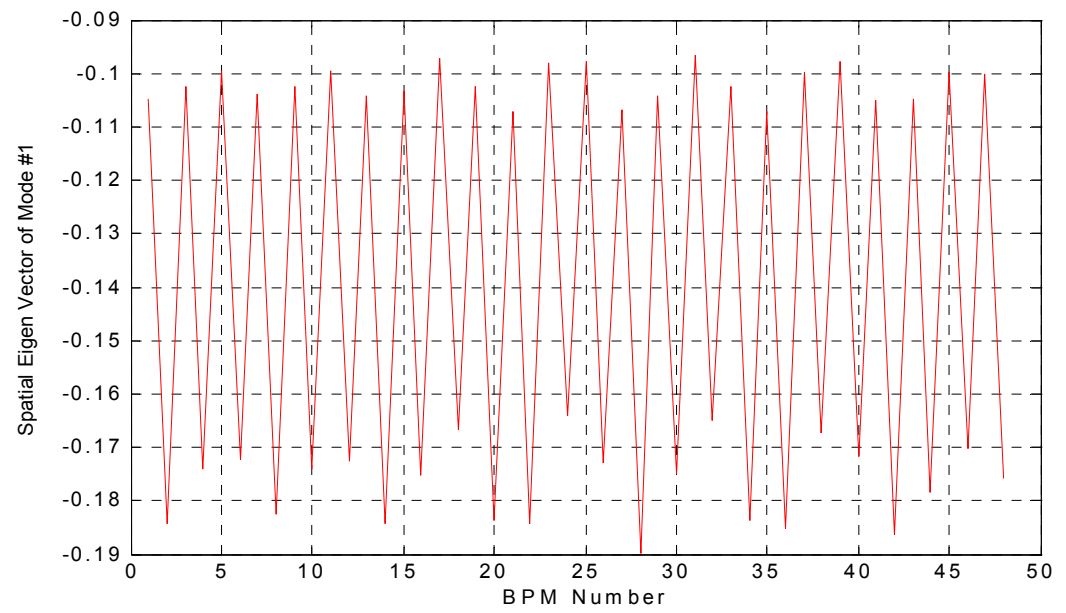

Fig. 1(d)

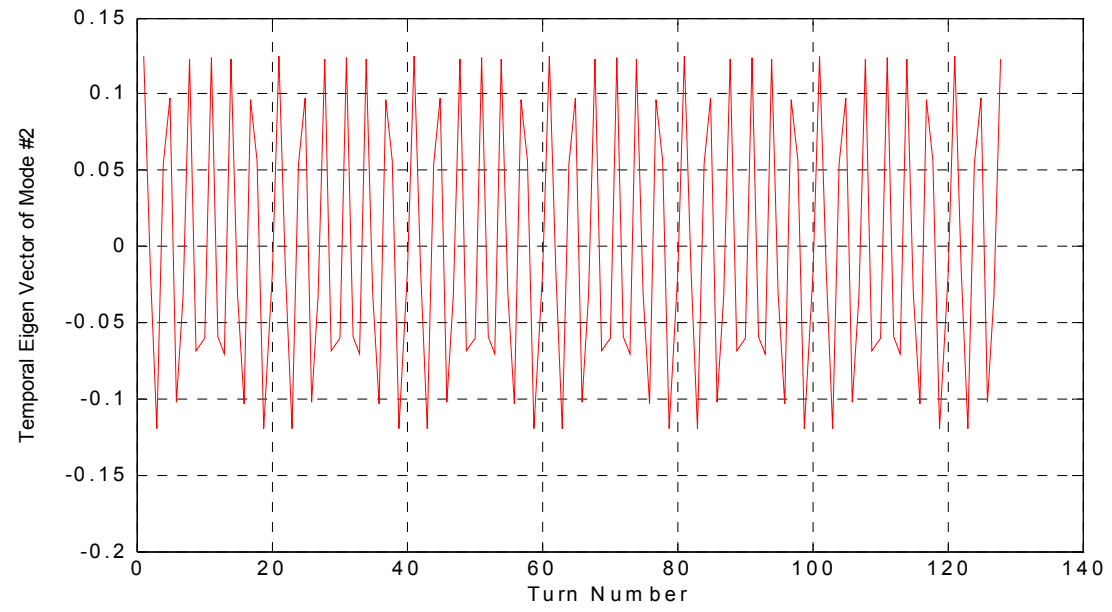

Fig. 1(e)

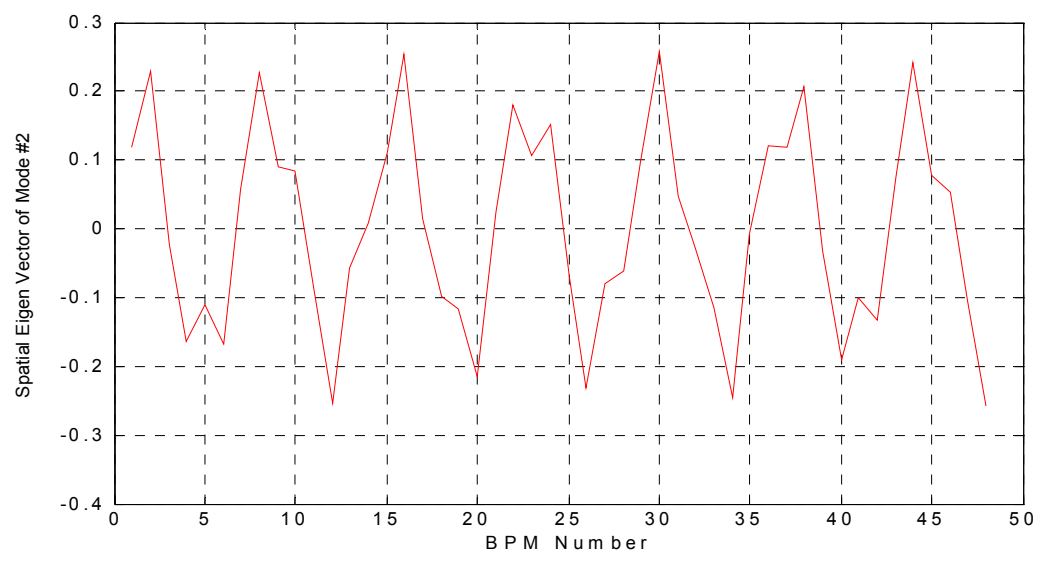

Fig. 1(f) 


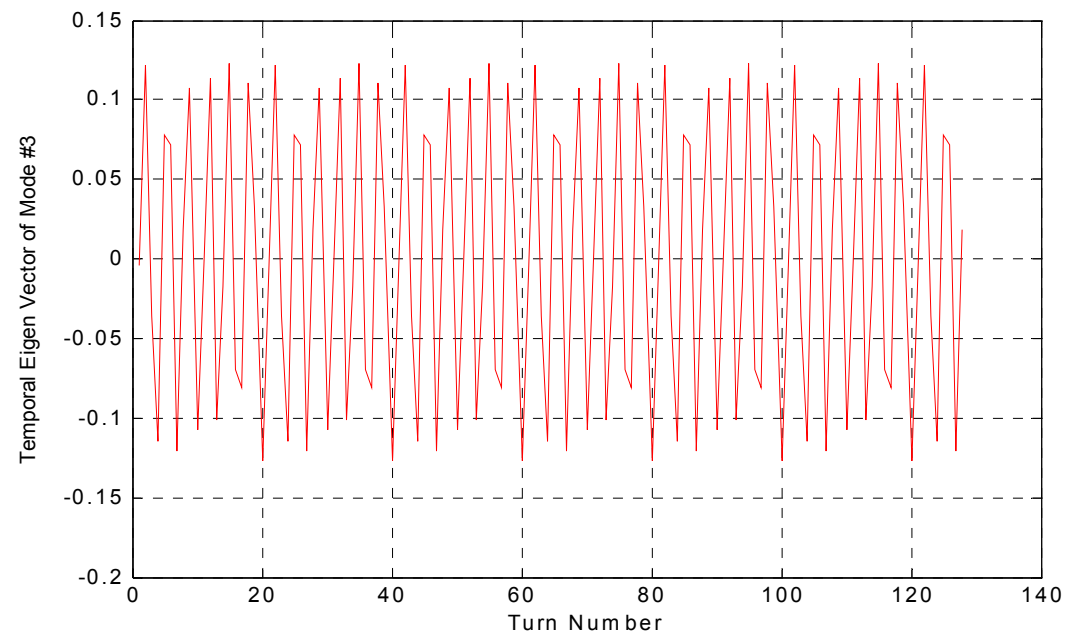

Fig. 1(g)

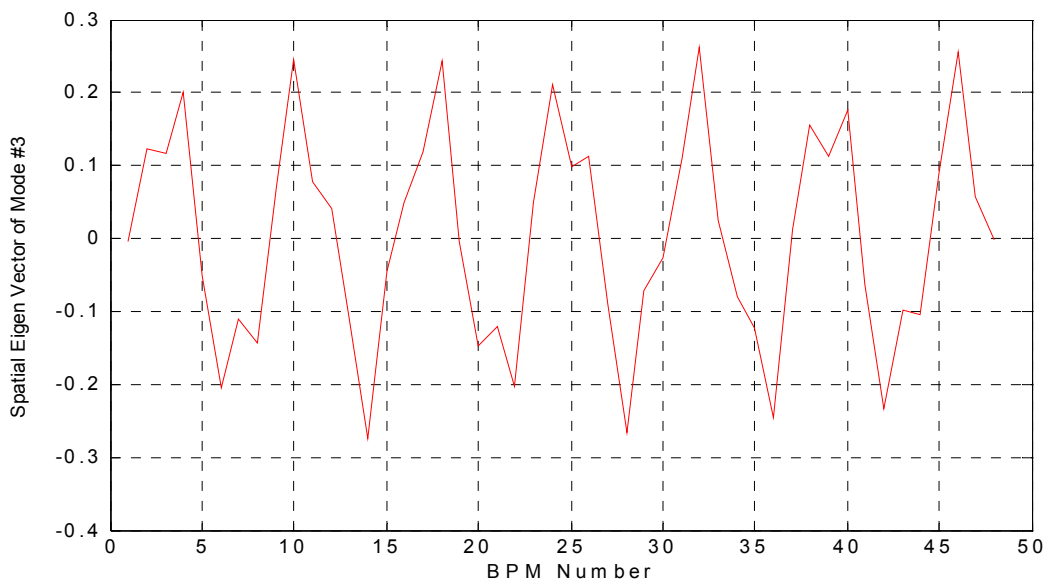

Fig. 1(h)

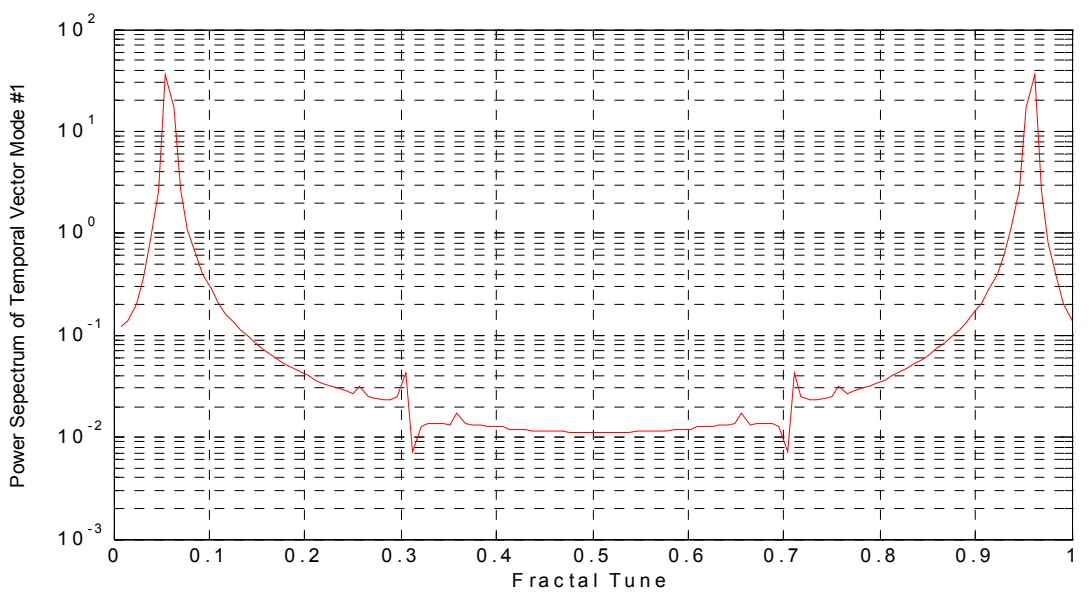

Fig. 1(i) 


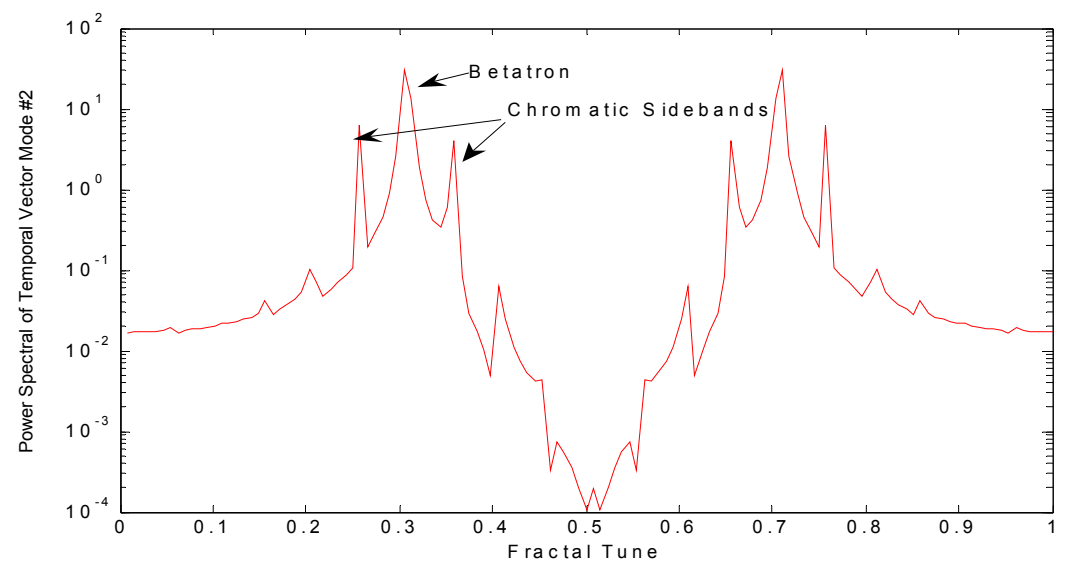

Fig. 1(j)

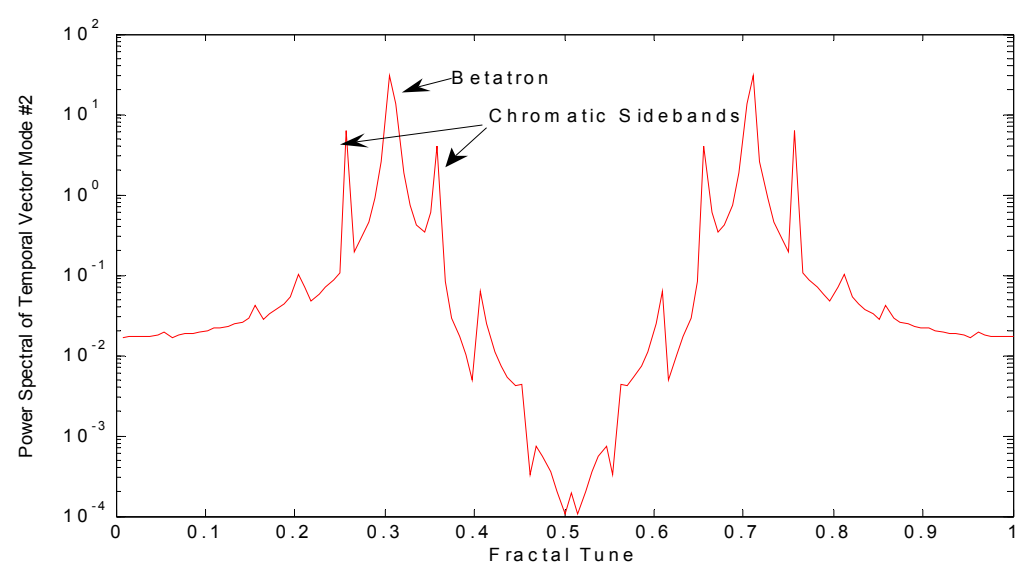

Fig. 1(k) 
Fig. 1(a) the $1^{\text {st }}$ turn and $128^{\text {th }}$ turn orbits calculated at the following conditions: the synchrotron tune $Q_{s}=0.05$, horizontal betatron tune $Q_{x}=6.7$, chromaticity $\xi=6$, after the beam was excited both transversely and longitudinally at the $0^{\text {th }}$ turn with a 1-mrad angle kick at the $1^{\text {st }} \mathrm{BPM}$ and momentum offset $\delta=0.005$.

Fig. 1(b) eigen values of all the modes.

Fig. 1(c) the temporal eigen vector of mode 1.

Fig. 1(d) the spatial eigen vector of mode 1.

Fig. 1(e) the temporal eigen vector of mode 2.

Fig. 1(f) the spatial eigen vector of mode 2.

Fig. $1(\mathrm{~g})$ the temporal eigen vector of mode 3.

Fig. 1(h) the spatial eigen vector of mode 3 .

Fig. 1(i) the unnormalized power spectrum of the temporal vector of mode 1.

Fig. 1(j) the unnormalized power spectrum of the temporal vector of mode 2.

Fig. 1(k) the unnormalized power spectrum of the temporal vector of mode 3. 


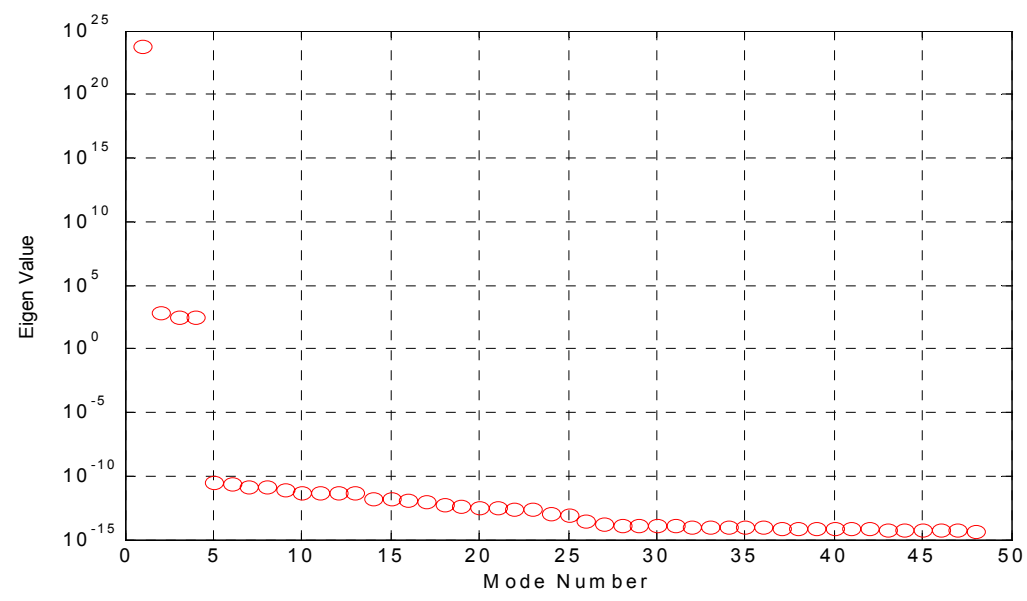

Fig. 2(a)

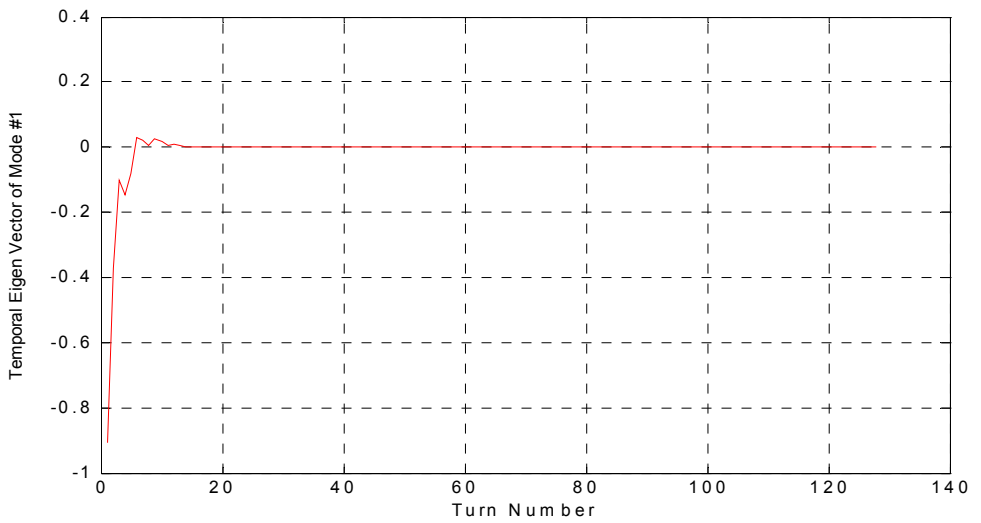

Fig. 2(b)

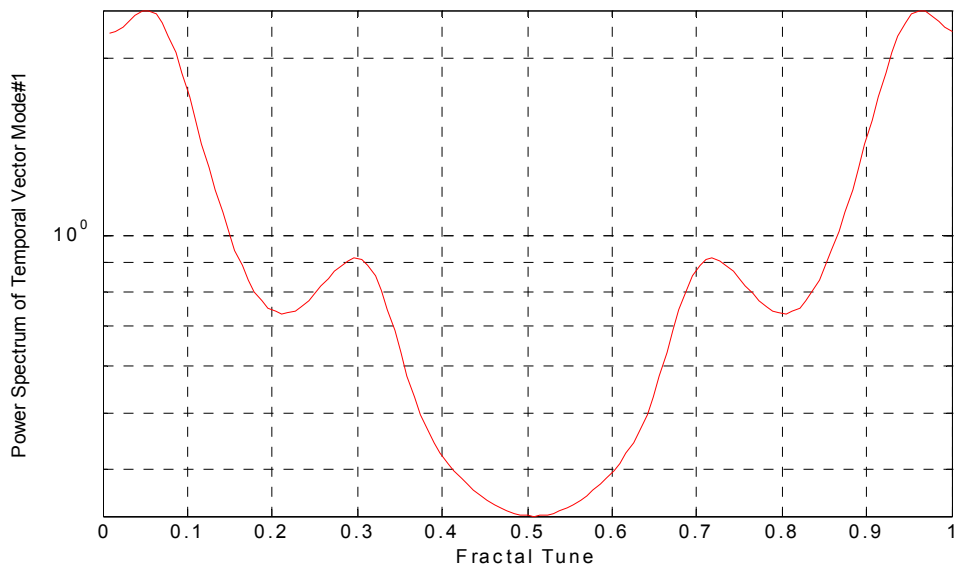

Fig. 2(c) 


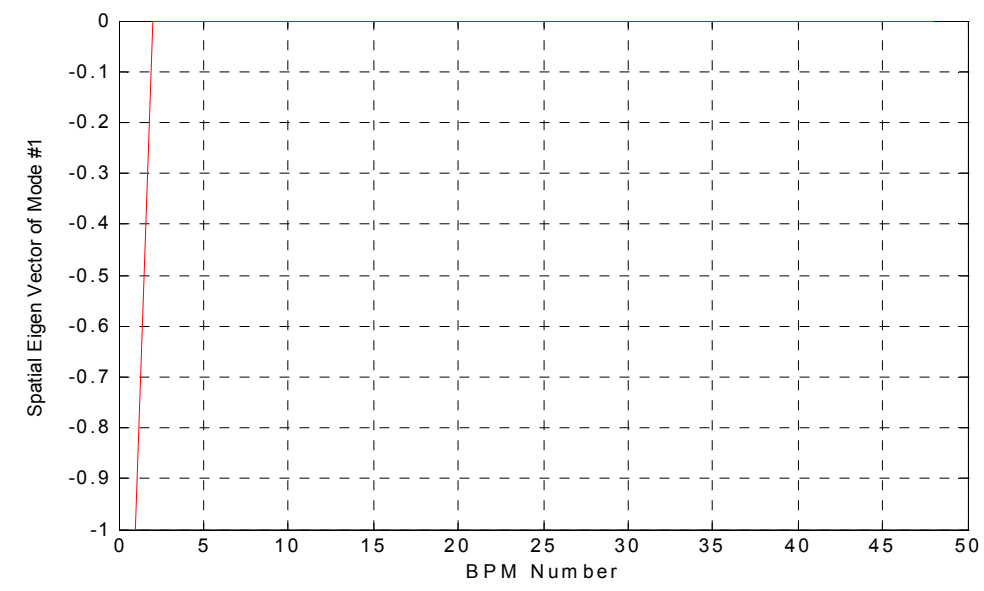

Fig. 2(d)

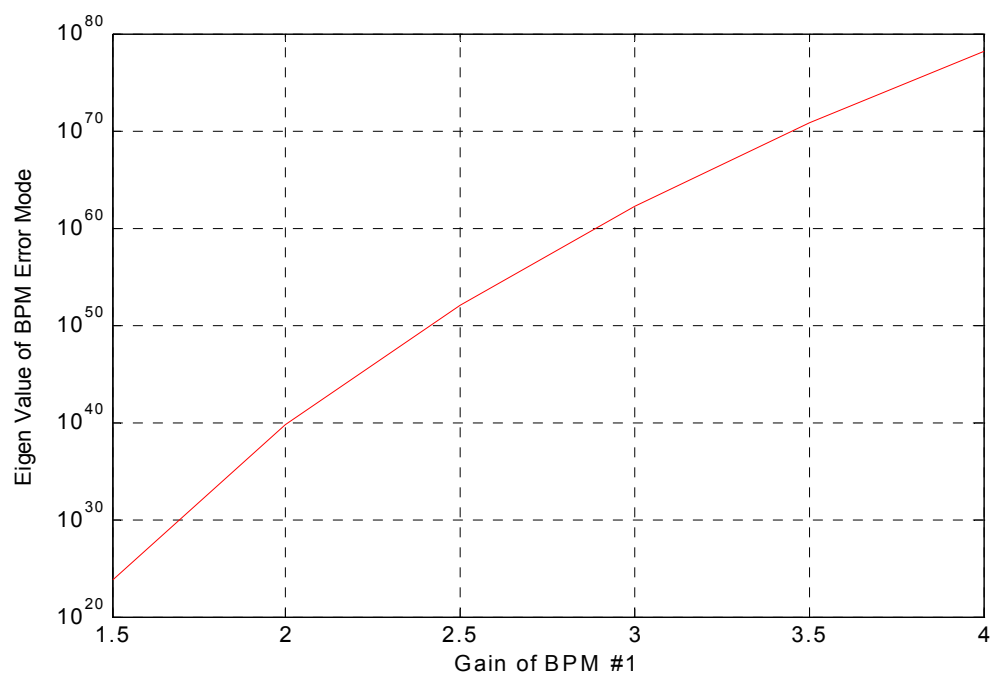

Fig. 2(e)

Fig. 2(a) set a different gain at 1.5 for the $1^{\text {st }}$ BPM while keeping other conditions the same with Fig.1, eigen values of all the modes.

Fig. 2(b) the temporal eigen vector of mode 1 .

Fig. 2(c) the unnormalized power spectrum of the temporal vector of mode 1 .

Fig. 2(d) the spatial eigen vector of mode 1 .

Fig. 2(e) the eigen value of the BPM error mode $v s$. the gain of the $1^{\text {st }}$ BPM. 


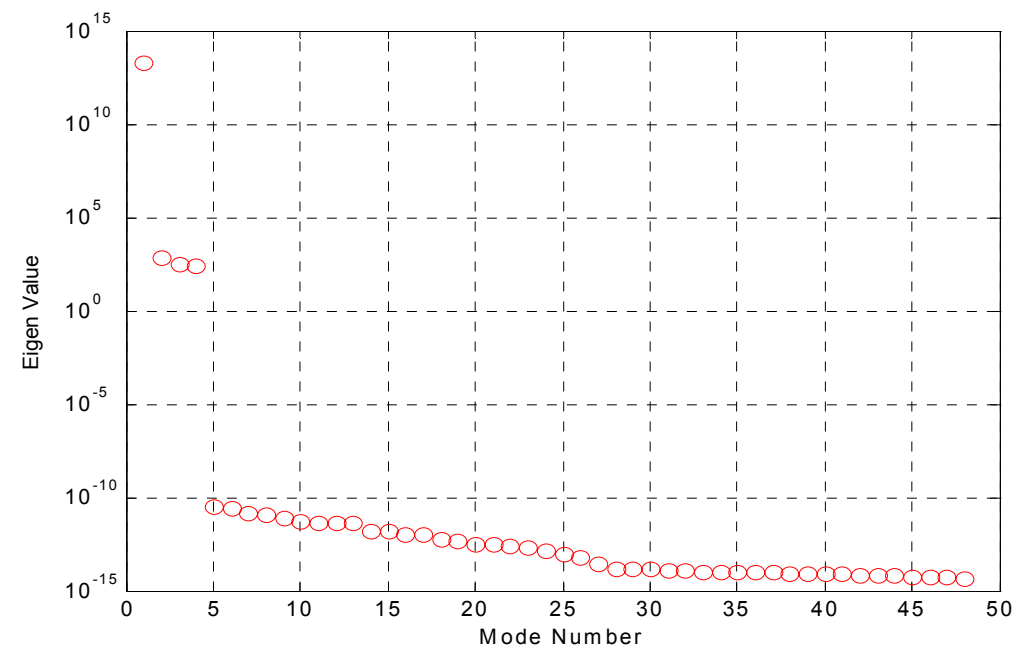

Fig. 3(a)

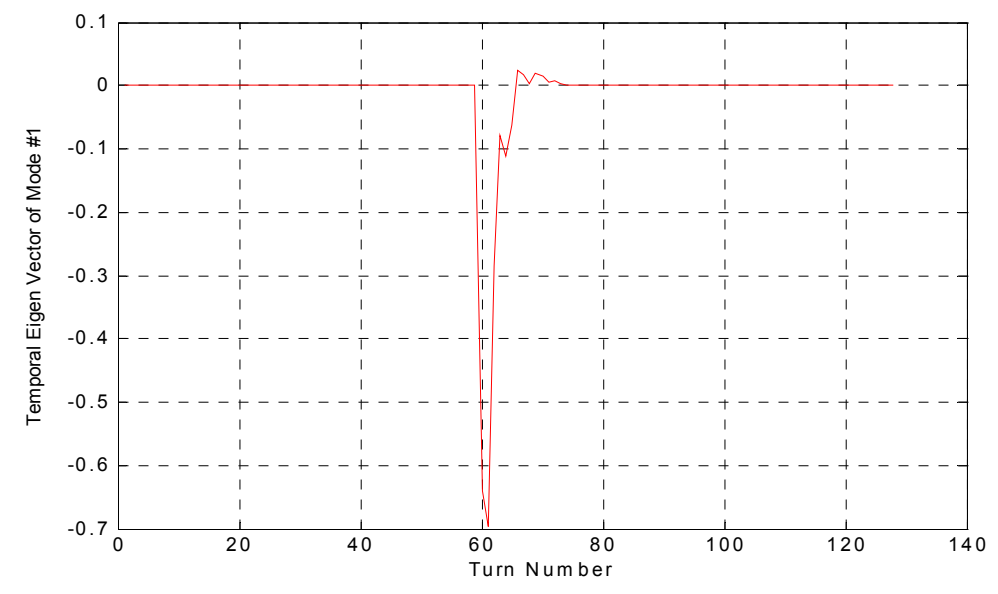

Fig. 3(b)

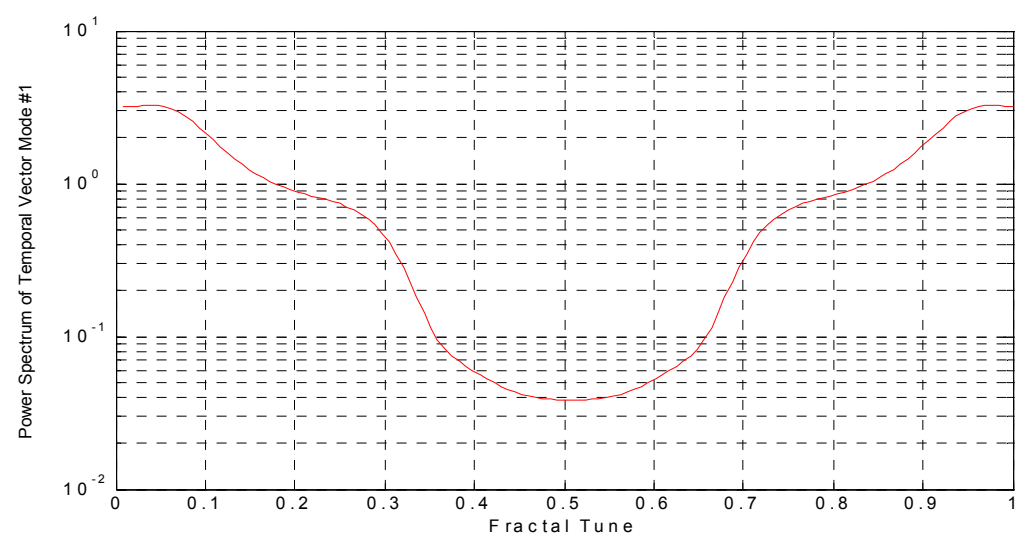

Fig. 3(c) 


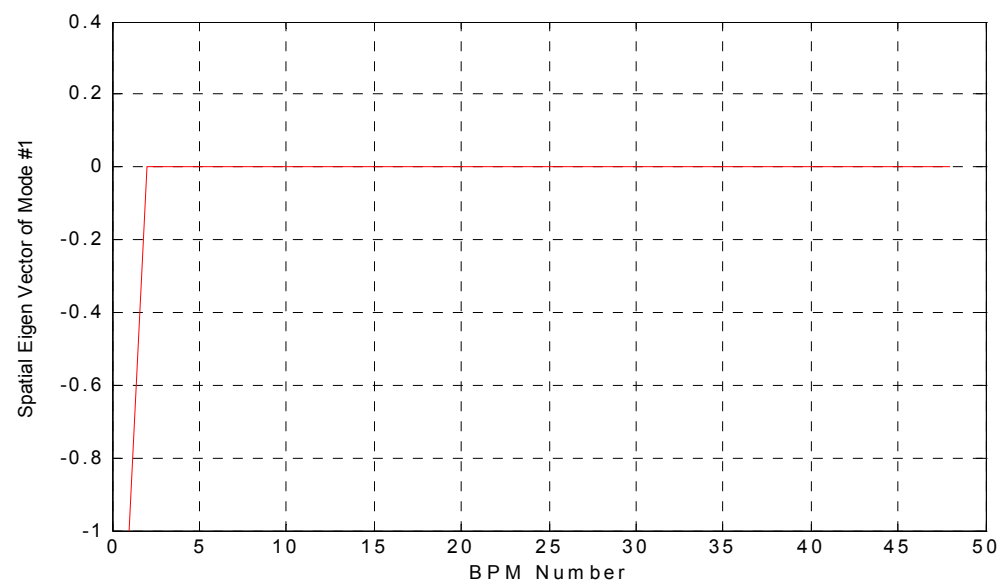

Fig. 3(d)

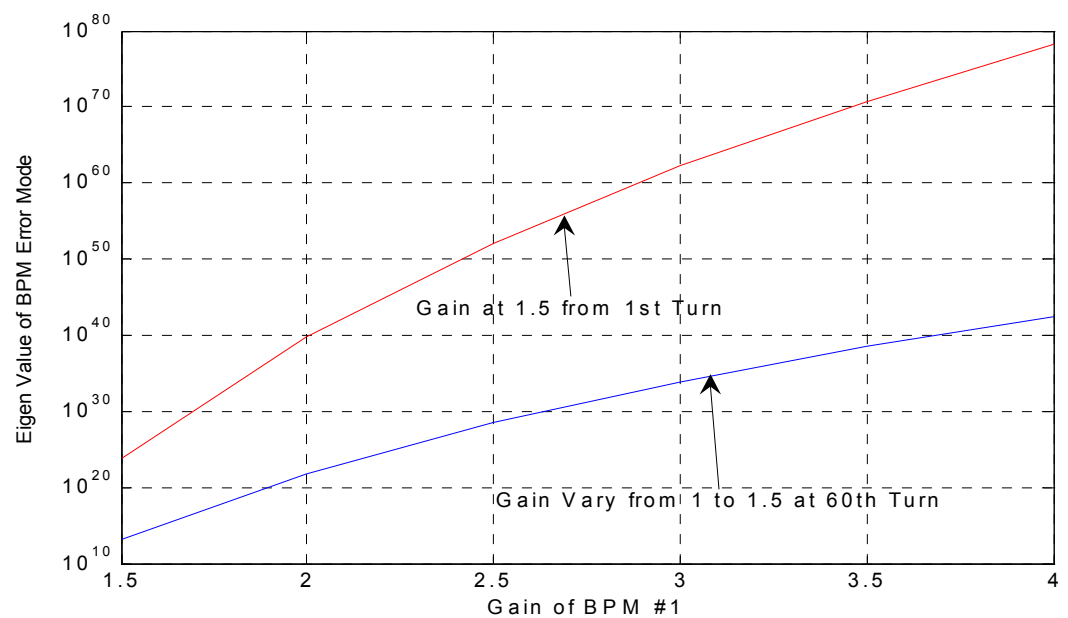

Fig. 3(e)

Fig. 3(a) change the gain since the $60^{\text {th }}$ turn from 1 to 1.5 for the $1^{\text {st }}$ BPM while keeping other conditions the same with Fig.1, eigen values of all the modes.

Fig. 3(b) the temporal eigen vector of mode 1.

Fig. 3(c) the unnormalized power spectrum of the temporal vector of mode 1 .

Fig. 3(d) the spatial eigen vector of mode 1.

Fig. 3(e) the eigen value of the BPM error mode $v s$. the gain of the $1^{\text {st }}$ BPM. The blue curve represents the different gain of the $1^{\text {st }}$ BPM starts at the $60^{\text {th }}$ turn, and the red curve starts at the $1^{\text {st }}$ turn. 